\title{
BioxLab: Laboratório de Informática em Saúde - Bioxthica
}

\author{
Alexandre Fonseca Brandão \\ Universidade Estadual de Campinas - UNICAMP \\ Instituto Brasileiro de Neurociências e de Neurotecnologia - BRAINN \\ BioxLab - https://www.brainn.org.br/inovacao/propriedade-intelectual/\#bioxtech-br \\ abrandao@ifi.unicamp.br
}

\begin{abstract}
Resumo: Sistemas de Realidade Virtual (RV) têm sido utilizados na ciência como ferramentas de ensino e aprendizagem, formação técnica e entretenimento. No campo da reabilitação neurofuncional, a RV oferece uma oportunidade real para complementar e estimular a terapia convencional em pessoas que convivem com limitações físicas e cognitivas. Pertencente ao BRAINN (um Cepid FAPESP), o BioxLab consiste em um laboratório de design, testes e aprimoramento de soluções de Informática em Saúde para aplicações em recuperação motora e neurofuncional. Estas aplicações são controladas a partir dos movimentos captados por um dispositivo de reconhecimento de gestos (de desenvolvimento próprio) que consiste em um sonar (ultrassom) e Arduino ou pelo Microsoft Kinect (dispositivo comercial) que digitaliza o corpo do usuário e cria coordenadas espaciais de suas articulações, permitindo a interação gestual.
\end{abstract}

Palavras-chave: reconhecimento corporal; realidade virtual; recuperação neurofuncional; dispositivos vestíveis.

\section{Introdução}

O Instituto Brasileiro de Neurociências e de Neurotecnologia - BRAINN [1] foi criado em 2013 como um Centro de Pesquisa, Inovação e Difusão da FAPESP [2], está sediado na Faculdade de Ciências Médicas da Universidade Estadual de Campinas UNICAMP, e busca desenvolver novos métodos e técnicas voltadas à prevenção, diagnóstico, reabilitação e tratamento de doenças cerebrais debilitantes, especialmente a epilepsia e o acidente vascular cerebral (AVC).

Entre os projetos orientados à criação e testes de novas tecnologias para neuroreabilitação, há uma linha de pesquisa voltada à realidade virtual (RV) e recuperação neurofuncional, que é responsável por três áreas complementares: 1 . desenvolvimento de dispositivos vestíveis para o reconhecimento corporal e controle de ambientes virtuais (a partir de interação gestual); 2. softwares de análise do movimento e; 3. aplicativos para interação dos pacientes com ambientes virtuais imersivos e não imersivos voltados ao contexto da reabilitação física e neurofuncional.

Neste contexto, foi estruturada a Bioxthica (Spionoff do BRAINN) e o BioxLab - Laboratório de Informática em Saúde da Bioxthica (figura 1), designado a liderar projetos de inovação nas áreas de informática em saúde e a interagir com Clínicas de Reabilitação, Centros de Saúde e Institutos de Ciência e Tecnologia (ICTs) para a transferência de tecnologia, capacitação de profissionais (especialistas em cinesiologia) e na colaboração em projetos científicos $[3,4]$.

O BioxLab é coordenado pelo Dr. Alexandre Brandão, Pesquisador Associado do BRAINN e do Instituto de Física Gleb Wataghin (IFGW) da UNICAMP.

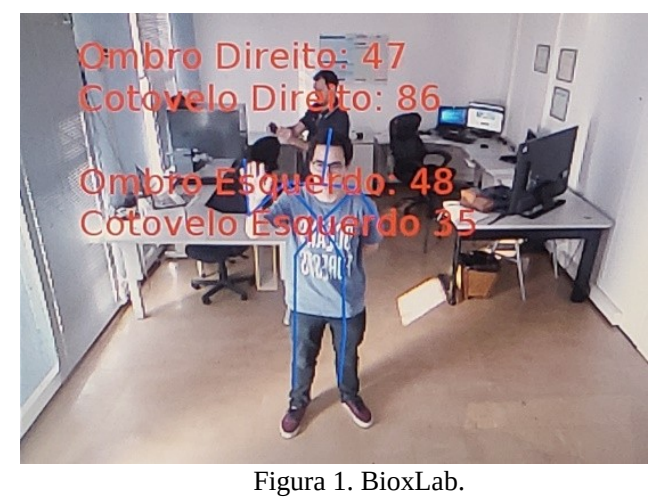

\section{Projetos e contribuições tecnológicas e científicas \\ Os projetos do laboratório estão subdivididos em 4 competências:}

2.1 Desenvolvimento de software para o registro e mensuração de amplitude do movimento (ADM) [5,6]

$\mathrm{O}$ software (figura 2) oferece uma opção aos especialistas da área da saúde acerca do estudo do movimento humano (cinesiologia), representa uma alternativa de baixo custo sendo um recurso de avaliação não invasivo.

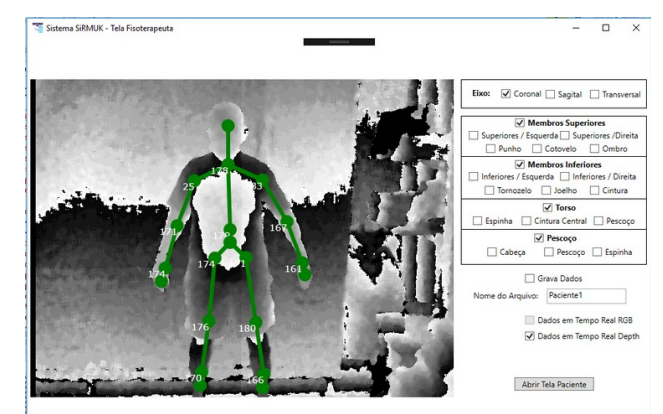

Figura 2. Interface do software de mensuração e registro de ADM. 


\subsection{Desenvolvimento de aplicativos de realidade virtual} imersiva e não imersiva

A interação gestual do usuário com o ambiente de RV ocorre de forma intuitiva, fisicamente ativa e essencialmente lúdica, por meio de gestos motores prédeterminados para cada aplicativo. O reconhecimento destes gestos, que permite esta interação gestual com o ambiente de RV, ocorre em duas etapas: $1^{\circ}$ ) o corpo de uma pessoa deve ser identificado por um sensor que o distingue dos demais objetos presentes no mesmo ambiente; $2^{\circ}$ ) os segmentos corporais, da pessoa identificada, devem ser agora atribuídos de características que possam servir de controle a um sistema computacional.

Os aplicativos GestureCollection (figura 3) oferecem ao usuário/paciente uma forma de interação fisicamente ativa, com controle motor a partir dos membros superiores e inferiores [7-11].
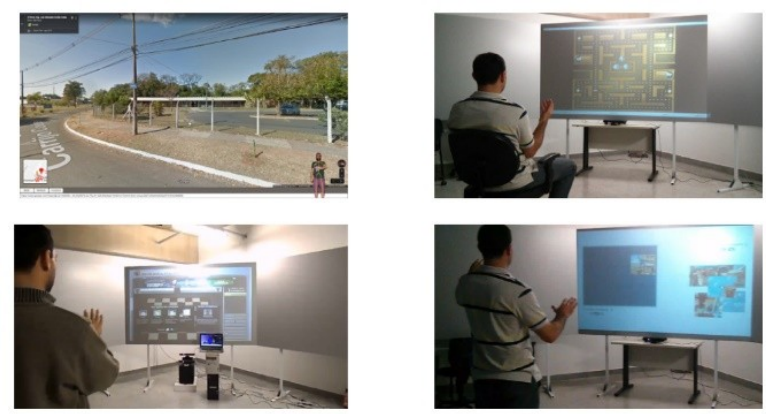

Figura 3. Interface dos aplicativos (não imersivos) GestureCollection.

Os aplicativos e-House e e-Street (figura 4), simulam um ambiente residencial e urbano, respectivamente, e foram modelados a partir do motor de jogo Unity3D, com o propósito de oferecer aos usuários desafios de navegação (orientação espacial) e simulação de situações de risco (prevenção de quedas e travessia de ruas) [12].
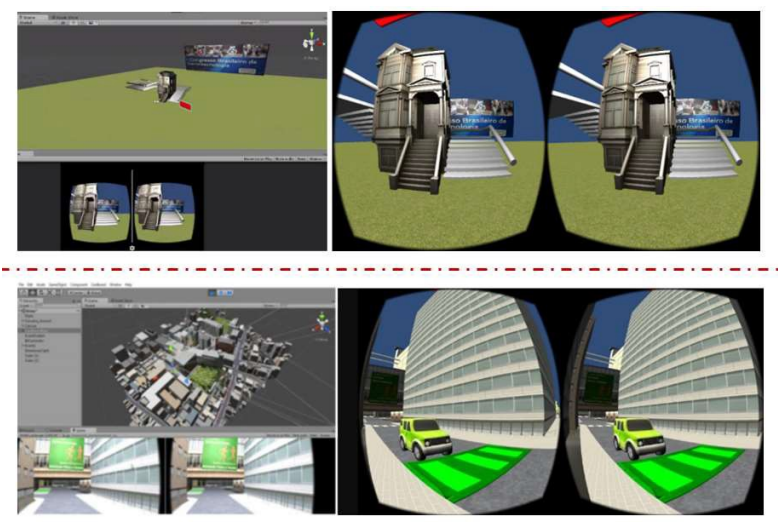

Figura 4. Interface e ambiente de desenvolvimento dos aplicativos (imersivos) e-House (imagem superior) e e-Street (imagem inferior).
2.3 Desenvolvimento de dispositivos vestíveis para Interação Humano-Computador (IHC) a partir de interação gestual

Para controlar o ambiente virtual a partir dos movimentos de marcha estacionária, foi construído um dispositivo (hardware) a partir de sensores ultrassom (sonar) conectados fisicamente (wired) à uma placa controladora (Arduino); os sinais de entrada consistem da diferença de altura $(\Delta S$, considerando a posição inicial S0) do sonar, e enviados ao Arduino (figura 5). O Arduino envia o sinal (via bluetooth) para o software que está sendo executado em um smartphone (inserido em óculos de RV), o qual permite o controle de navegação (por interação gestual) dos ambientes eHouse e e-Street pelo usuário [13,14].

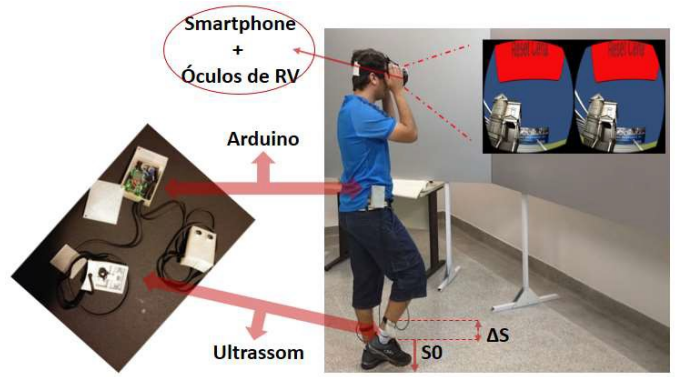

Figura 5. Dispositivo de interação para marcha estacionária.

2.4 Aplicação das tecnologias desenvolvidas em testes de usabilidade com voluntários/pacientes em terapias de recuperação motora e neurofuncional

A fim de evidenciar o aumento de conectividade cerebral durante a interação com as soluções descritas anteriormente, foram realizados exames de Imagem de Ressonância Magnética funcional - fMRI (do inglês, functional Magnetic Resonance Image), que consiste em uma técnica de neuroimagem, não invasiva, utilizada para encontrar áreas cerebrais ativas mediante a execução de diferentes tarefas (motoras ou cognitivas) ou mesmo quando o indivíduo está em repouso (restingstate).

Para estudar a conectividade cerebral (figura 6), são analisadas as relações entre as diversas áreas cerebrais durante experimentos de estado de repouso (restingstate fMRI), os quais são realizados antes e após a interação com um ou mais dos aplicativos [15].
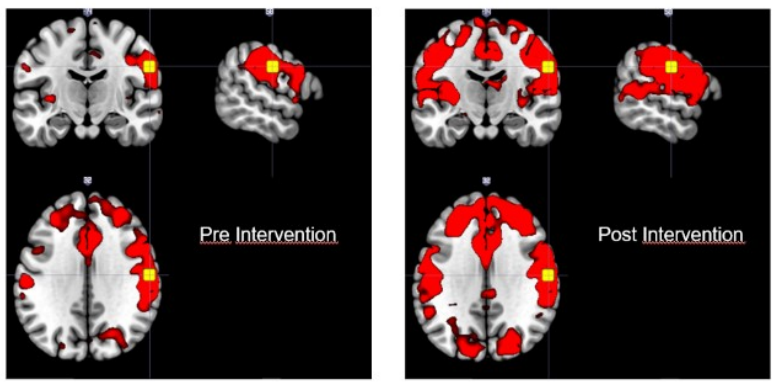

Figura 6. Mapa de correlação da atividade cerebral antes a após a interação com os aplicativos não-imersivos. 


\section{Parceiros}

São parceiros do BioxLab, pesquisadores das ICTs:

- UNICAMP: Prof ${ }^{a}{ } r^{a}$ Gabriela Castellano, Instituto de Física Gleb Wataghin (IFGW) | Prof. Dr. Li Li Min e $\mathrm{Dr}^{\mathrm{a}}$ Sara Regina Meira de Almeida, Faculdade de Ciências Médicas (FCM) | Prof ${ }^{a} \operatorname{Dr}^{\mathrm{a}}$ Paula Teixeira Fernandes, Faculdade de Educação Física (FEF) | Dr. Thomas Beltrame, Instituto de Computação (IC); * publicações em colaboração [57,11-16].

- USP: Prof. Dr. Cláudio Fabiano Motta Toledo, Instituto de Ciências Matemáticas e de Computação (ICMC); * publicações em colaboração [17].

- UFSJ: Prof. Dr. Diego Roberto Colombo Dias, Departamento de Computação (DComp); * publicações em colaboração [5,6,7,12,13,14].

- UNESP: Prof. Dr. José Remo Ferreira Brega, Departamento de Computação;

- UNIFESP: Prof. Dr. Marcelos de Paiva Guimarães, Reitoria/UAB; * publicações em colaboração $[6,7,12,14,18]$.

- UFES: Prof ${ }^{\mathrm{a}}$ Dr $^{\mathrm{a}}$ Ester Miyuki Nakamura Palacios, Centro Biomédico, Departamento de Ciências Fisiológicas;

- UFOP: Prof ${ }^{a}$ Dr $^{\mathrm{a}}$ Gilda Aparecida de Assis, Departamento de Computação; * publicações em colaboração $[15,19,20]$.

- UFSCAR: Prof ${ }^{a} \operatorname{Dr}^{\mathrm{a}}$ Daniela Godoi Jacomassi, Departamento de Educação Física e Motricidade Humana (DEFMH) $\mid$ Prof $^{\mathrm{a}} \mathrm{Dr}^{\mathrm{a}}$ Paula Regina Mendes da Silva Serrão, Prof Dr Maurício Jamami e Prof. Dr. Nivaldo Antônio Parizotto, Departamento de Fisioterapia (DFisio) | Prof. Dr. Daniel Marinho Cezar da Cruz, Departamento de Terapia Ocupacional (DTO) $\mid$ Prof. Dr. Luis Carlos Trevelin, Departamento de Computação (DC); * publicações em colaboração [5,7,8,9,10,11,12,13].

\section{Agradecimentos}

Fundação de Amparo à Pesquisa do Estado de São Paulo - FAPESP (Processo n 2015/03695-5).

\section{Bibliografia}

[1] BRAINN (2020) Instituto Brasileiro de Neurociências e de Neurotecnologia. https://www.brainn.org.br/

[2] Cepid FAPESP (2020) Centro de Pesquisa, Inovação e Difusão da FAPESP. http://cepid.fapesp.br/centro/11/

[3] BIOXTHICA (2017) Unidade EMBRAPII CPQD e Bioxthica desenvolvem projeto com foco na reabilitação de pessoas com deficiências motoras. https://www.cpqd.com.br/noticias/unidade-embrapii-cpqde-bioxthica-desenvolvem-projeto-com-foco-nareabilitacao-de-pessoas-com-deficiencias-motoras/

[4] BIOXTHICA (2020) Virtual Reality applied to neurorehabilitation.

https://parque.inova.unicamp.br/en/virtual-reality-appliedto-neurorehabilitation/

[5] Brandão, A.F. et al. (2016) RehabGesture: an alternative tool for measuring human movement. Telemedicine and $e-$ Health Journal 22(7). DOI: 10.1089/tmi.2015.0139
[6] Godoy, S. et al. (2020) Apoio à Reabilitação de Pacientes Utilizando Dispositivo de Reconhecimento de Gestos. Revista Ibérica de Sistemas e Tecnologias de Informação 28(4): 204-216.

[7] Brandão, A.F. et al (2018) GestureCollection for Motor and Cognitive Stimuli: Virtual Reality and e-Health prospects. Journal on Health Informatics 10(1): 9-16.

[8] Frade, M.C.M. et al. (2019) Reproducibility and validity of the six-minute stationary walk test associated with virtual reality in copd subjects. Respiratory Care 64(4): 425-433. DOI: $10.4187 /$ respcare.06237

[9] Tossini et al. (2017) Influência da realidade virtual sobre a dor, fadiga, capacidade funcional e qualidade de vida na fibromialgia: estudo de caso. Acta Fisiátrica 24(4): 212215. 10.5935/0104-7795.20170039

[10] Krutli, R.S. et al. (2018) Applicability and evaluation of the GestureChair virtual game: comparison between people with and without spinal cord injury. SBC Journal on Interactive System 9: 64-71.

[11] Sporkens-Magna, T. et al. (2020) Intervenção por Realidade Virtual e Exercício Físico em Idosos. Journal of Health Informatics, 12(3): 77-82 2020.

[12] Dias, DRC. et al. (2018) eStreet: Virtual Reality and Wearable Devices Applied to Rehabilitation. In: Gervasi O. et al. (eds) Computational Science and Its Applications - ICCSA 2018. ICCSA 2018. Lecture Notes in Computer Science 10963. Springer. DOI: 10.1007/978-3-319-95171$\underline{360}$

[13] Brandão, A.F. et al. (2019) e-Street for Prevention of Falls in the Elderly: An Urban Virtual Environment for HumanComputer-Interaction from Lower Limb Movements. In: Iano Y., Arthur R., Saotome O., Vieira Estrela V., Loschi H. (eds). Proc. 3rd Brazilian Technology Symposium. BTSym 2017. Springer. DOI: 10.1007/978-3-319-93112$\underline{8 \quad 25}$

[14] Brandão, A.F. et al. (2020) Biomechanics Sensor Node for Virtual Reality: A Wearable Device Applied to Gait Recovery for Neurofunctional Rehabilitation. Lecture Notes in Computer Science, 2020. Best Paper Award In: XX International Conference on Computer Science and Applications (ICCSA). (Ahead of Print)

[15] Brandão, A.F. et al. (2019) Gestural Interaction applied to upper-limb motor improvement. SBC Journalon Interactive Systems 10(1).

[16] Brandão A.F., Almeida S.E.M. (2017) Controlar o Computador só com a Mente?! (Cap. 11); Livro: Neuro o Quê?! Neurociência! A Ciência e a Arte do Cérebro (Li Li Min - Org.), $2^{\text {a }}$ ed. Campinas: ADCiência.

[17] Jurioli, M. M. et al. (2020) Wearable Device for Immersive Virtual Reality Control and Application in Upper Limbs Motor Rehabilitation. Lecture Notes in Computer Science. (Ahead of Print)

[18] Viana, C. et al. (2020) Blockchain para gerenciamento de prontuários eletrônicos. Revista Ibérica de Sistemas e Tecnologias de Informação 28(4): 177-187.

[19] Assis, G.A. et al. (2019) Evaluation of a Protocol for fMRI Assessment Associated with Augmented Reality Rehabilitation of Stroke Subjects. SBC Journal on Interactive Systems 10(1).

[20] Assis, G.A. et al. (2017) Electromyography and Augmented Reality for Motor Rehabilitation. In: Proc. 19th Symp on Virtual and Augmented Reality. DOI 10.1109/SVR.2017.14 